\title{
The Comparison of the Classical Keplerian Orbit Elements, Non-Singular Orbital Elements (Equinoctial Elements), and the Cartesian State Variables in Lagrange Planetary Equations with $J_{2}$ Perturbation: Part I
}

\author{
Jung Hyun Jo ${ }^{1 \dagger}$, In Kwan Park ${ }^{1}$, Nammi Choe ${ }^{2}$, and Mansoo Choi ${ }^{1}$ \\ ${ }^{1}$ Korea Astronomy and Space Science Institute, Daejeon 305-348, Korea \\ ${ }^{2}$ Korea Aerospace Research Institute, Daejeon 305-333, Korea
}

Two semi-analytic solutions for a perturbed two-body problem known as Lagrange planetary equations (LPE) were compared to a numerical integration of the equation of motion with same perturbation force. To avoid the critical conditions inherited from the configuration of LPE, non-singular orbital elements (EOE) had been introduced. In this study, two types of orbital elements, classical Keplerian orbital elements (COE) and EOE were used for the solution of the LPE. The effectiveness of EOE and the discrepancy between EOE and COE were investigated by using several near critical conditions. The near one revolution, one day, and seven days evolutions of each orbital element described in LPE with COE and EOE were analyzed by comparing it with the directly converted orbital elements from the numerically integrated state vector in Cartesian coordinate. As a result, LPE with EOE has an advantage in long term calculation over LPE with $\mathrm{COE}$ in case of relatively small eccentricity.

Keywords: Lagrange planetary equation, equinoctial elements, $J_{2}$ perturbation, numerical integration

\section{INTRODUCTION}

The motion of the satellite around the Earth can be expressed as a simple Newtonian equation in the Earth centered coordinates. The two bodies rotating around each other without any external forces can be described by the exact mathematical solution. We generally call this simple analytically solvable problem a two-body problem. However, the motion of the satellite in real environment is also dictated by the gravitational and non-gravitational forces due to the irregular shape of the Earth, other celestial bodies, the Earth's atmosphere, Solar wind, and planetary radiation, etc. These forces other than the point mass gravitational force are called perturbation forces. Even before Classical Dynamics and Mathematics were well defined, some of giants in the science left a big footprint in describing the satellite (planetary) motion with the perturbation (Broucke \& Cefola 1972, Taff 1985).

Several methods to calculate the evolution of the satellite's orbit governed by the perturbation were discovered since the Principia of Newton. The Lagrange and Gauss planetary equations (LPE and GPE) are two most recognized methods to describe the change rates of orbital elements with the partials of the disturbing forces. With these theories, Kozai (1959) and Brouwer \& Clemence (1961) derived the semi-analytical solutions for the short period, long period, and secular perturbations with the variety of mathematical techniques.

With the recent development of the modern computer, many of precision orbit propagation tasks have been processed with direct numerical integration (DNI) of the

(c) This is an Open Access article distributed under the terms of the Creative Commons Attribution Non-Commercial License (http://creativecommons.org/licenses/by-nc/3.0/) which permits unrestricted non-commercial use, distribution, and reproduction in any medium, provided the original work is properly cited.

Received Oct 09, 2010 Revised Dec 10, 2010 Accepted Dec 17, 2010 ${ }^{\dagger}$ Corresponding Author

E-mail:jhjo39@kasi.re.kr

Tel: +82-42-865-3238 Fax: +82-42-861-5610 
Newtonian equation of motion as the convention of the Cowell's orbit integration method. The DNI method has achieved one centimeter level of precision orbit propagation (Luthcke et al. 2003). One day arc to even over ten years arc of satellite's orbit can be propagated by the DNI method to be estimated for the analysis of the perturbations (Nerem et al. 1993). However, the degradation of orbit propagation due to the integration and numerical errors is inevitable as the integration time span grows. Also, the computation time increases as the requested integration time span is getting inflated. To avoid the several inconvenient factors mentioned previously, the analytic or semi-analytic orbit propagators are still investigated and applied in many Celestial Mechanics and Orbital Mechanics problem.

As the development of a domestic satellite orbit propagator and estimator Korea Astronomy and Space Science Institute Orbit propagator and Estimator (KASIOPEA) is right on track (Jo 2008), the characteristic figures of the equations of motion, invariants, and primary variables to be considered must be investigated.

In this study, two types of LPE orbital solutions with classical Keplerian orbital elements (COE) and EOE were reviewed. Before the effectiveness of EOE and the discrepancy between EOE and COE were investigated by using several near critical conditions, a result from DNI had been tested for a reference by the two-body analytic solution. Two types of solution and integration were performed for three different lengths of time. The 13,000 seconds, one day, and seven days evolutions of each orbital element described in LPE were analyzed by comparing it with the directly converted orbital elements from the numerically integrated state vector in Cartesian coordinate.

\section{LAGRANGE PLANETARY EQUATION WITH CLASSICAL ORBITAL ELEMENTS}

The general theory for finding the rates of change of the osculating elements has been known as the LPE of motion, or simply the Lagrangian variation of parameters (Vallado 2004). In two-body problem it is confirmed that the position and velocity components (in Cartesian coordinates) at any instant permit the determination of a unique set of six COE. And these COE do not change with time. However, most of problems we encountered in the satellite motion have the relatively larger acceleration from the primary body and perturbing accelerations from the others. The equation of the motion of a satellite may be put in a form of Cartesian rectangular coordinates: $(\ddot{x}, \ddot{y}, \ddot{z})$. It is a system of differential equations of the sixth order. Also, these equations of the rectangular coordinates of the elliptic motion can be expressed in terms of the time and six constants (integrals) of integration. As a result, obtained six equations, each of the first order, are exactly equivalent to the original three equations, each of the second order (Brouwer \& Clemence 1961). With the six COE from these equations, the equations of the variation of parameters with respect to the small conservative disturbing forces can be obtained as Eqs. (1-6).

$\frac{d a}{d t}=\frac{2}{n a} \frac{\partial R}{\partial M}$

$\frac{d e}{d t}=\frac{1-e^{2}}{n a^{2} e} \frac{\partial R}{\partial M}-\frac{\sqrt{1-e^{2}}}{n a^{2} e} \frac{\partial R}{\partial \omega}$

$\frac{d i}{d t}=\frac{\cos i}{n a^{2} \sqrt{1-e^{2}} \sin i} \frac{\partial R}{\partial \omega}-\frac{1}{n a^{2}\left(1-e^{2}\right)^{1 / 2} \sin i} \frac{\partial R}{\partial \Omega}$

$\frac{d \omega}{d t}=-\frac{\cos i}{n a^{2} \sqrt{1-e^{2}} \sin i} \frac{\partial R}{\partial i}+\frac{\sqrt{1-e^{2}}}{n a^{2} e} \frac{\partial R}{\partial e}$

$\frac{d \Omega}{d t}=\frac{1}{n a^{2} \sqrt{1-e^{2} \sin i}} \frac{\partial R}{\partial i}$

$\frac{d M}{d t}=n-\frac{1-e^{2}}{n a^{2} e} \frac{\partial R}{\partial e}-\frac{2}{n a} \frac{\partial R}{\partial a}$

where,
$a$ semi-major axis
$e$ eccentricity of orbit
$i$ inclination of orbital plan
$\omega$ argument of perigee
$M$ mean anomaly
$\Omega$ ascending node
$n$ mean motion
$R$ disturbing function

Using this LPE, we can investigate the perturbation characteristics of specific orbital element with the specific perturbing force. In our case, the time dependent changes of the orbital elements due to the $J_{2}$ zonal nonspherical harmonic gravitational perturbation are analyzed. For Earth gravity perturbations, the disturbing function is merely the negative of the disturbing part of geo-potential (Blitzer 1975). The disturbing function can be described as in Eq. (7).

$$
R=-\frac{\mu J_{2}}{r}\left(\frac{R_{e}}{r}\right)^{2} P_{2}(\sin \phi)
$$


where,

$\mu$ gravitational constant multiplied by the mass of the Earth

$=3.986004418 \times 10^{14}\left(\mathrm{~m}^{3} / \mathrm{sec}^{2}\right)$

(Vallado 2004)

$r$ radius of satellite

$\phi$ latitude of the sub-satellite point

$R_{2}$ radius of the Earth

$P_{2}$ Legendre polynomial degree 2

$$
=\frac{1}{2}\left(3 \sin ^{2} \phi-1\right)
$$

However, the disturbing function, $R$ is not a function of COE. The partial derivatives of $R$ in Eqs. (1-6) should survive only when $R=R(a, e, i, \omega, M, \Omega)$. The $r$ and $\sin \phi$ can be substituted with the functions of $a, e, i, v, \omega$ as in Eqs. (8) and (9).

$$
\begin{gathered}
r=\frac{a\left(1-e^{2}\right)}{1+e \cos v} \\
\sin \phi=\sin i \sin (v+\omega)
\end{gathered}
$$

where,

$v$ true anomaly

With the perturbation by the $J_{2}$ term, only the first order secular and the short period disturbing function are survived (Kozai 1959). After several variable substitutions, averaging, and first order approximation, Kozai (1959) and Brouwer \& Clemence (1961) successfully solved the variation of equation, Eqs. (1-6). The solutions for short period and secular terms derived by Kozai (1959) are presented in Appendix A.

\section{LAGRANGE PLANETARY EQUATION WITH EQUINOCTIAL ORBITAL ELEMENTS}

With the COE in LPE, there can be some inconvenience with specific types of orbits. As you can see in Eqs. (2-6), small eccentricities or small inclinations can make the equation unstable. The eccentricity and the $\sin i$ in the denominators of the equations may become significant trouble with very small value. The Many efforts have been made to avoid this inconvenience by selecting new set of orbital elements. Some elements are functions of the classical elements, $e, \omega$, and $\Omega$, others are functions of $i$ and $\Omega$. Note that the elements $f$ and $g$ in Eqs. (11) and (12) have been discussed in Moulton (1914), Brouwer \& Clemence (1961), Cohen \& Hubbard (1962), and Walker et al. (1985) but that $h$ and $k$ differ slightly by authors.
Arsenault et al. (1970) introduced the name 'equinoctial elements' for this type of non-singular elements. In this study, a set of equinoctial elements introduced by Walker et al. (1985) shown in Eqs. (10-15) were used for the simulation.

$$
\begin{aligned}
& p=a\left(1-e^{2}\right) \\
& f=e \cos (\Omega+\omega) \\
& g=e \sin (\Omega+\omega) \\
& L=M+\Omega+\omega \\
& h=\tan \left(\frac{i}{2}\right) \cos (\Omega) \\
& k=\tan \left(\frac{i}{2}\right) \cos (\Omega)
\end{aligned}
$$

The transformation from the equinoctial elements to the COE is given in Eqs. (16-21).

$$
\begin{aligned}
& a=\left(\frac{p}{1-f^{2}-g^{2}}\right) \\
& e=\sqrt{f^{2}+g^{2}} \\
& i=2 \tan ^{-1}\left(\sqrt{h^{2}+k^{2}}\right) \\
& \Omega=\tan ^{-1}\left(\frac{k}{h}\right) \\
& \omega=\tan ^{-1}\left(\frac{g}{f}\right)-\tan ^{-1}\left(\frac{k}{h}\right) \\
& M=L-\tan ^{-1}\left(\frac{g}{f}\right)
\end{aligned}
$$

With the calculation of Lagrange bracket with the EOE and the differentiation of Eqs. (10-15) with respect to time, the derivatives of EOE can be found in terms of COE and their derivatives. Eqs. (1-6) can be replaced by the new set of LPE, Eqs. (22-27).

$$
\begin{aligned}
\frac{d p}{d t}= & 2 \sqrt{\frac{P}{\mu}}\left(-g \frac{\partial \tilde{R}}{\partial f}+f \frac{\partial \tilde{R}}{\partial g}+\frac{\partial \tilde{R}}{\partial L}\right) \\
\frac{d f}{d t}= & \frac{1}{\sqrt{\mu p}}\left[2 p g \frac{\partial \tilde{R}}{\partial p}-\left(1-f^{2}-g^{2}\right) \frac{\partial \tilde{R}}{\partial g}\right. \\
& \left.-\frac{g s^{2}}{2}\left(h \frac{\partial \tilde{R}}{\partial h}+k \frac{\partial \tilde{R}}{\partial k}\right)+\{f+(1+w) \cos L\} \frac{\partial \tilde{R}}{\partial L}\right]
\end{aligned}
$$




$$
\begin{aligned}
\frac{d g}{d t}= & \frac{1}{\sqrt{\mu p}}\left[-2 p f \frac{\partial \tilde{R}}{\partial p}+\left(1-f^{2}-g^{2}\right) \frac{\partial \tilde{R}}{\partial f}\right. \\
& \left.+\frac{f s^{2}}{2}\left(h \frac{\partial \tilde{R}}{\partial h}+k \frac{\partial \tilde{R}}{\partial k}\right)+\{g+(1+w) \sin L\} \frac{\partial \tilde{R}}{\partial L}\right] \\
\frac{d L}{d t}= & \sqrt{\mu p}\left(\frac{w}{p}\right)^{2}+\frac{s^{2}}{2 \sqrt{\mu p}}\left(h \frac{\partial \tilde{R}}{\partial k}+k \frac{\partial \tilde{R}}{\partial k}\right) \\
\frac{d h}{d t}= & \frac{s^{2}}{2 \sqrt{\mu p}}\left\{h\left(g \frac{\partial \tilde{R}}{\partial f}-f \frac{\partial \tilde{R}}{\partial g}-\frac{\partial \tilde{R}}{\partial L}\right)-\frac{s^{2}}{2} \frac{\partial \tilde{R}}{\partial k}\right\} \\
\frac{d k}{d t}= & \frac{s^{2}}{2 \sqrt{\mu p}}\left\{k\left(g \frac{\partial \tilde{R}}{\partial f}-f \frac{\partial \tilde{R}}{\partial g}-\frac{\partial \tilde{R}}{\partial L}\right)+\frac{s^{2}}{2} \frac{\partial \tilde{R}}{\partial h}\right\}
\end{aligned}
$$

where

$s^{2}=1+h^{2}+k^{2}$

$w=\frac{p}{r}=1+f \cos L+g \sin L$

The disturbing function, Eq. (7) can be rewritten with the substitution of Eqs. (30) and (31).

$$
r=\frac{p}{w}
$$

$\sin \phi=\frac{2(h \sin L-k \cos L)}{s^{2}}$

New set of derivatives of the disturbing functions in terms of EOE is shown in Eqs. (32-37).

$$
\begin{aligned}
& \frac{\partial \tilde{R}}{\partial p}=\frac{3 \mu}{2 w r^{2}} J_{2}\left(\frac{R_{e}}{r}\right)^{2}\left\{\frac{12(h \sin L-k \cos L)^{2}}{s^{4}}-1\right\} \\
& \frac{\partial \tilde{R}}{\partial f}=-\frac{3 \mu \cos L}{2 w r} J_{2}\left(\frac{R_{e}}{r}\right)^{2}\left\{\frac{12(h \sin L-k \cos L)^{2}}{s^{4}}-1\right\} \\
& \frac{\partial \tilde{R}}{\partial g}=-\frac{3 \mu \sin L}{2 w r} J_{2}\left(\frac{R_{e}}{r}\right)^{2}\left\{\frac{12\left((h \sin L-k \cos L)^{2}\right.}{s^{4}}-1\right\} \\
& \frac{\partial \tilde{R}}{\partial L}=-\frac{2 \mu}{r s^{2}}(h \cos L+k \sin L) J_{2}\left(\frac{R_{e}}{r}\right)^{2} P_{2}^{\prime}(\sin \phi) \\
& \quad-\frac{3 \mu}{r w}(g \cos L-f \sin L) J_{2}\left(\frac{R_{e}}{r}\right)^{2} P_{2}(\sin \phi) \\
& \frac{\partial \tilde{R}}{\partial h}=-\frac{2 \mu}{r s^{4}}\left\{\left(1-h^{2}+k^{2}\right) \sin L+2 h k \cos L\right\} J_{2}\left(\frac{R_{e}}{r}\right)^{2} P_{2}^{\prime}(\sin \phi) \\
& \frac{\partial \tilde{R}}{\partial k}=\frac{2 \mu}{r s^{4}}\left\{\left(1+h^{2}-k^{2}\right) \cos L+2 h k \sin L\right\} J_{2}\left(\frac{R_{e}}{r}\right)^{2} P_{2}^{\prime}(\sin \phi)
\end{aligned}
$$

where

$$
\begin{aligned}
P_{2}^{\prime}(\sin \phi) & =\frac{3}{1-\sin ^{2} \phi}\left\{\frac{1}{2} \sin \phi\left(3 \sin ^{2} \phi-1\right)\right. \\
& \left.-\frac{1}{2}\left(5 \sin ^{3} \phi-3 \sin \phi\right)\right\}
\end{aligned}
$$

The description of the orbit evolution by the $J_{2}$ perturbation with EOE is completed.

\section{DIRECT NUMERICAL INTEGRATION OF THE EQUATION OF MOTION IN CARTESIAN COORDI- NATE}

A state vector and a force vector in Cartesian coordinate can be defined as in Eqs. (39) and (40)

$$
\vec{X}=\left[\begin{array}{c}
x \\
y \\
z \\
\dot{x} \\
\dot{y} \\
\dot{z}
\end{array}\right] \quad(39), \quad \dot{\vec{X}}=f(\vec{X})=\left[\begin{array}{c}
\dot{x} \\
\dot{y} \\
\dot{z} \\
-\mu \frac{x}{r^{3}} \\
-\mu \frac{y}{r^{3}} \\
-\mu \frac{z}{r^{3}}
\end{array}\right]
$$

where

$$
r=\sqrt{x^{2}+y^{2}+z^{2}}
$$

The acceleration due to the $J_{2}$ zonal harmonic nonspherical gravitational perturbation also can be applied to the force vector as in Eq. (40). Point mass two-body gravitational acceleration and $J_{2}$ disturbing acceleration term both can be accumulated to form a total acceleration for the satellite.

$$
\overrightarrow{X_{J_{2}}}=f(\vec{X})_{J_{2}}=\left[\begin{array}{c}
0 \\
0 \\
0 \\
-\frac{3 \mu J_{2} R_{e}^{2} x}{2 r^{5}}\left(1-\frac{5 z^{2}}{r^{2}}\right) \\
-\frac{3 \mu J_{2} R_{e}^{2} y}{2 r^{5}}\left(1-\frac{5 z^{2}}{r^{2}}\right) \\
-\frac{3 \mu J_{2} R_{e}^{2} z}{2 r^{5}}\left(3-\frac{5 z^{2}}{r^{2}}\right)
\end{array}\right]
$$

where,

$J_{2}$ oblateness coefficient of the Earth $R_{e}$ radius of the Earth 
The equation of motion expressed in the Cartesian coordinate has an advantage in the numerical integration for its simplicity over analytic or semi-analytic type. And the perturbation functions and two-body equations do not have any singular point except $r=0$ (unusual condition with the finite size of the Earth). The straight forward formulation helps the design and the manipulation of external forces.

The transformation from the state vector to the COE can be achieved without any trouble in most elliptic case. The full procedure for this transformation can be found at Vallado (2004) and other references. Unlike semi-analytical solutions, this DNI method may introduce an error as time increases: truncation error. This type of error cannot be avoidable as the computer has limited digit of significant number and the numerical integration method inherits the approximation errors.

\section{NUMERICAL PROCEDURES}

In this study, the comparison of the results from the each solving method of the equation of satellite motion with $J_{2}$ perturbation is the primary objective. It is rather not possible to find any analytical exact solution to compare the each orbit. Remember the LPE type solution is a first order approximation by the variation of parameter.

First, four specific orbits chosen to represent particular orbit might introduce some inconvenience. The case I in the Table 1 represents an orbit with very small eccentricity and small sin $i$ terms; unusual equatorial low Earth orbit (LEO) satellite. The cases II and III show the typical condition of LEO satellite. The case IV displays an example of geodetic satellite. The COE initial conditions in the Table 1 can be transformed into Cartesian coordinates as in Table A1 in Appendix.

Second, the accuracy of the numerical integration of the equation of the motion with two-body force only in Cartesian coordinate was compared with the analytical

Table 1. Initial condition (Keplerian elements).

\begin{tabular}{lrrrrr}
\hline & I & II & III & IV & V \\
\hline$h_{0}(\mathrm{~km})$ & 360 & 550 & 1,350 & 5,500 & 360 \\
$e_{0}$ & 0.0005 & 0.005 & 0.05 & 0.1 & 0.005 \\
$i_{0}(\mathrm{deg})$ & 10.0 & 97.5 & 101.0 & 88.0 & 10.0 \\
$\Omega_{0}(\mathrm{deg})$ & 30.0 & 50.0 & 80.0 & 120.0 & 30.0 \\
$\omega_{0}(\mathrm{deg})$ & 30.0 & 30.0 & 30.0 & 30.0 & 30.0 \\
$v_{0}(\mathrm{deg})$ & 0.0 & 0.0 & 0.0 & 0.0 & 0.0 \\
\hline$h_{0}$ : altitude at perigee; Earth Radius: 6,378.137 km (Vallado 2004).
\end{tabular}

solution: mean motion on the orbital plane. To validate the DNI method, all cases of initial condition were tested. The value of $a, e, i, \omega, M_{0}, \Omega$ should not be changed as time advances in both solutions from analytic method and DNI method in two-body problem without any perturbation. However, in case of DNI method previously mentioned several errors will be accumulated on the state vector, as time advances or rather as we perform every step of numerical integration.

The difference (error) evolutions of orbital elements between the DNI method and the analytical solution are shown in Figs. 1-7. In case of DNI method, the integration time step must be chosen with careful consideration for classical Rung-Kutta 4th order method to minimize calculation load, round-off error, and truncation error. However, it is not a simple task to find an optimal integration step size for general cases.

In this study, fixed step sizes: 60 and 10 seconds were tested for all cases. As shown in Figs. 1 and 2, the accuracy was improved in 1 to 1,000 ratio as the time step was reduced in 6 to 1 ratio. With the integration step size of 60 seconds, error in the semi-major axis grows to about 200 meters for the case IV in 7 days. With the integration step size of 10 seconds, same orbit has only 0.025 meter error after 7 days. An optimal integration step size can be found with further test or an integration step size control can be applied to this study. However, 10 second step size was chosen for its obvious simplicity in decimal number with relatively acceptable accuracy.

The error in semi-major axis grows almost linearly as time advances for all four cases as shown in Fig. 2. The shorter period the orbit has (i.e. the orbit is closer to the

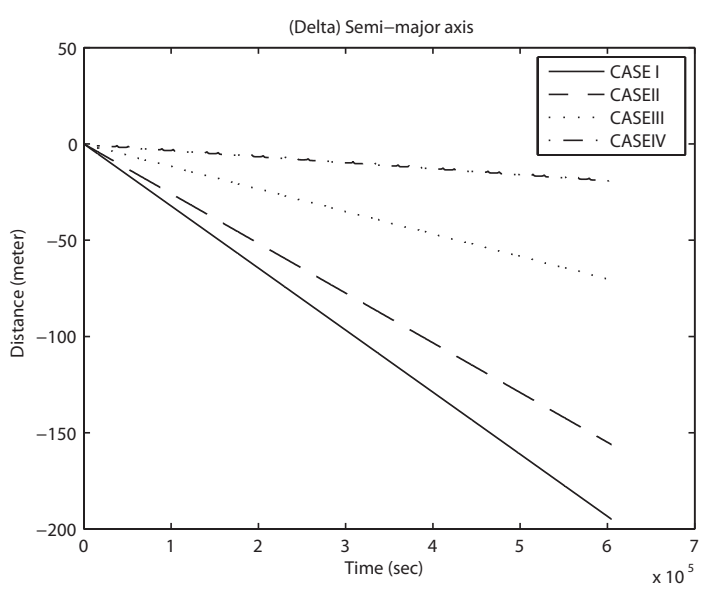

Fig. 1. The error evolution of semi-major axis from the state vector direct numerical integration method with integration step size: 60 seconds compared to the two-body analytical solution for seven days. 


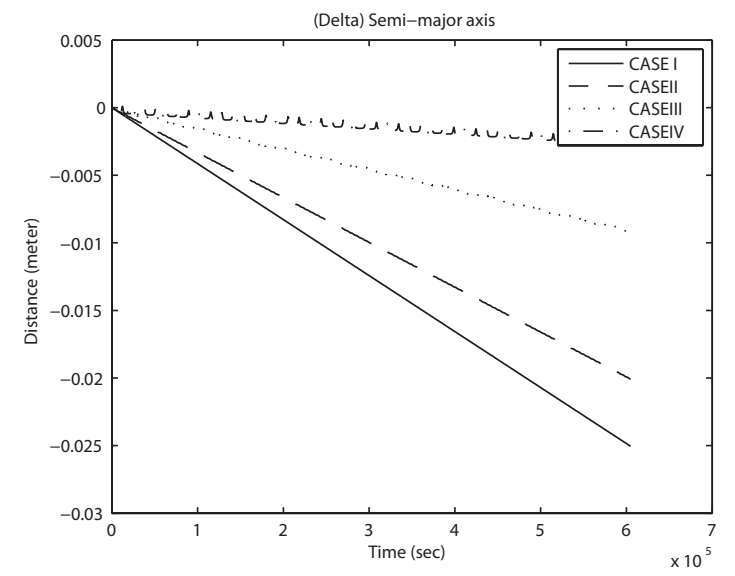

Fig. 2. The error evolution of semi-major axis from the state vector direct numerical integration method with step size: 10 seconds compared to the two-body analytical solution for seven days.

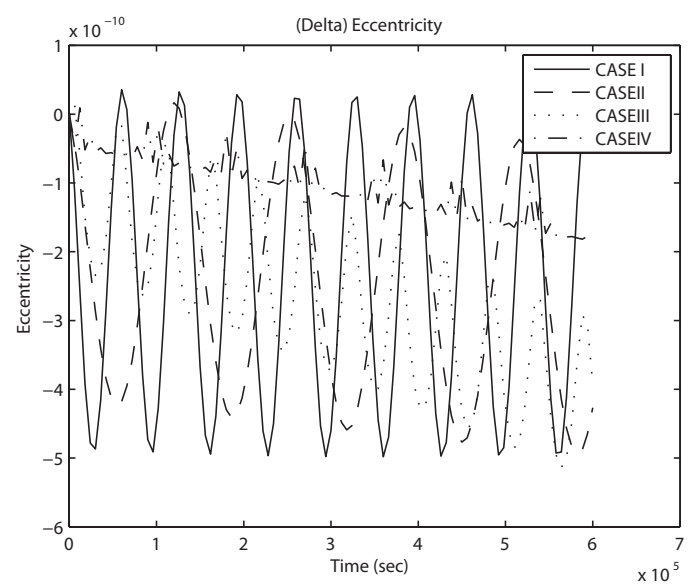

Fig. 3. The error evolution of eccentricity from the state vector direct numerical integration method compared to the two-body analytical solution for seven days.

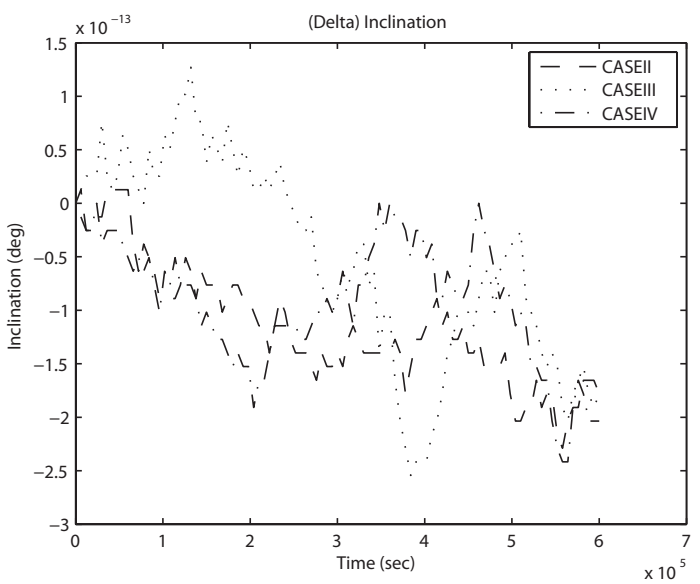

Fig. 4. The error evolution of inclination from the state vector direct numerical integration method compared to the two-body analytical solution for seven days.

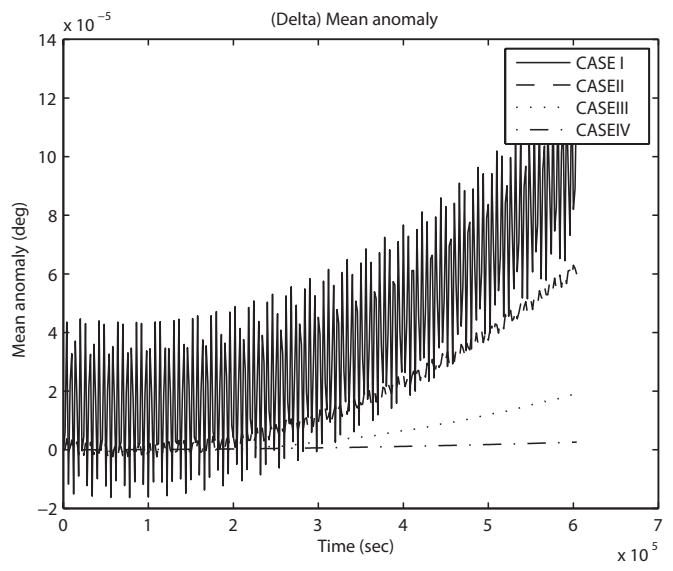

Fig. 5. The error evolution of mean anomaly at epoch from the state vector direct numerical integration method compared to the two-body analytical solution for seven days.

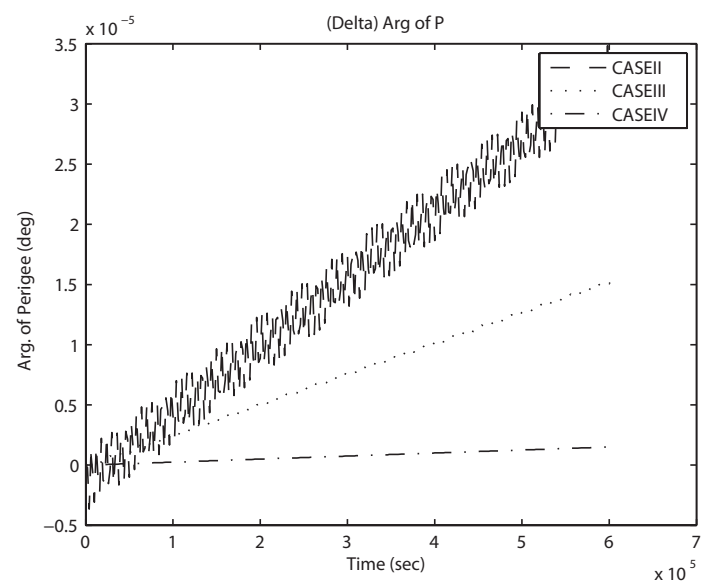

Fig. 6. The error evolution of the argument of perigee from the state vector direct numerical integration method compared to the two-body analytical solution for seven days.

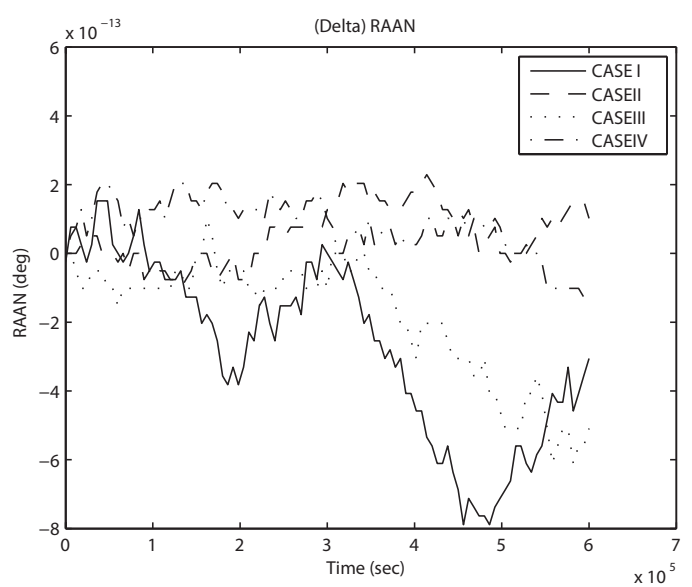

Fig. 7. The error evolution of the right ascension of ascending node from the state vector direct numerical integration method compared to the two-body analytical solution for seven days. 
Earth), the steeper slope the error shows. Since we used a fixed size integration step for all numerical integrations, the orbit with larger orbital period requires larger number of integration step to complete one revolution. It has benefits of smoother and dense derivatives, and shorter interval relative to the whole arc of orbit. Unlike other cases, the case IV shows the sinusoidal behavior with same period of orbital revolution

More complicated figures are shown in the error evolution in eccentricity for all cases as you can see in Fig. 3. The sinusoidal variation of each case shows same periodicity of orbital revolution. Over all tendencies are similar to those in semi-major axis case. However, the eccentricity looks more susceptible to the errors induced by the numerical integration.

The error evolutions of inclination and the right ascension of ascending node are shown in Figs. 4 and 7 respectively. In these two cases, it is hard to find any periodicity or obvious tendency except gradual degradation. The magnitude of the errors in both cases lies close to the lower bound of significant number of digit of the computer used in this study compare to the original values of both orbital elements.

The fast variable in the LPE, mean anomaly shows typical error evolution figures in Fig. 5. As shown in Fig. 6, the effect of error on the argument of perigee is stronger as shallower the ellipse is.

In summary, the deviations of the DNI method from the exact solution for two-body problem do not exceed $10^{-6}$ ratio in case of $e, 10^{-7}$ ratio in case of $M_{0}, \omega, 10^{-9}$ ratio in case of $a, 10^{-13}$ ratio in case of $i, \Omega$ for 7 days. For the comparison of LPE with two different sets of orbital elements and state variable DNI, we tested the DNI with two-body exact solution first. In conclusion, state variable with $J_{2}$ perturbation DNI can be used as a reference to compare two general perturbation method using $\mathrm{COE}$ and EOE with the confidence level shown in Figs. 2-7.

Third, the general perturbation solutions by using COE and EOE shown in Appendix A and B are calculated and the set of state variable with $J_{2}$ perturbation was integrated by a classical Runge-Kutta method with 10 second integration time step. Though the characteristics of the two-body DNI is shown by comparing it with the analytic solution in the previous section, the two-body problem with $J_{2}$ perturbation cannot be expected to act in same manner. However, the $J_{2}$, a dominant perturbation term by mass displacement from the perfect sphere is still 1:1,000 ratio to point mass two-body gravitational force.

\section{COMPARISON RESULTS AND DISCUSSION}

The time evolution of each orbital element (whether it is integral or variable) by three different approach for solution was investigated with the initial conditions suggested in the previous section. The initial condition of orbits to be investigated was chosen to check the system instability caused by the near singular condition in the denominators of the equations.

The first comparison test with 13,000 seconds duration, which represents one revolution of the longest period of test orbit (case IV), is expected to show major characteristics of short period perturbation as $J_{2}$ term only produce short period and secular perturbation (Kozai 1959, Brouwer \& Clemence 1961). The evolution of 6 COE and 6 EOE compared to the DNI results for the case II are presented in Fig. 8. Most of elements agree to those from other methods but eccentricity. The calculation of increment in eccentricity with COE LPE leads to unstable behavior in case of relatively small eccentricity $\left(\sim 10^{-3}\right)$. The saw tooth shaped trace of mean anomaly is mainly due to the mean motion (Fig. 8).

The difference evolution in semi-major axes of two semi-analytic solutions from the DNI result is shown in Fig. 9. The gaps at the epoch $(t=0)$ in Fig. 9 were produced by the off-set due to an addition of first solution from the LPE with COE. Even application of mean value to adjust osculating orbital elements to mean orbital elements (Not mean orbit) cannot get rid of these gaps completely. The arbitrary initial orbit elements as shown in Table 1 are used for orbit simulation in this study, however, we obtain an osculating orbital element from the calculation of the Lagrange planetary equation's solution with perturbing forces. The result from LPE with perturbing forces is always an osculating orbit. In other words, using a priori orbit elements, perturbed orbit must not be admitted to use at next epoch. This can be adopted both COE and EOE case. In the DNI case, a numerical integration of dynamics including perturbations produces an osculating orbit at any epoch naturally.

As we have mentioned, LPE uses input elements as a reference orbit and calculates each element's variation caused by perturbations. In this study, orbit simulation of each case has been set a same initial condition, so same orbit elements can be used as an initial value for input at each case, COE, EOE, and DNI. The difference evolution of right ascension of ascending node for all cases is shown in Fig. 10. The inclination and the ascending node decide the spatial position of an orbital plane in an inertial frame. The trends of the evolutions of the inclination 
and the right ascension of ascending node are very similar naturally. As shown in Fig. 11a, the amplitude of error variation in argument of perigee is relatively larger than any other angle related orbital elements. However, the error of mean anomaly in the case I by COE LPE shows unreasonable behavior compared to the results from other method. For the analysis of this type of error in the case I, the initial condition of the case $V$ was selected. The initial condition of both cases has identical components except eccentricity. The eccentricity of the case $\mathrm{V}$ is exactly ten times of that of the case I. As the eccentricity in the case I was 0.0005 , both terms in the right hand side of Eq. (2) induced some inconveniency. On the contrary, the one in the case $\mathrm{V}$ with the value of 0.005 did not introduce

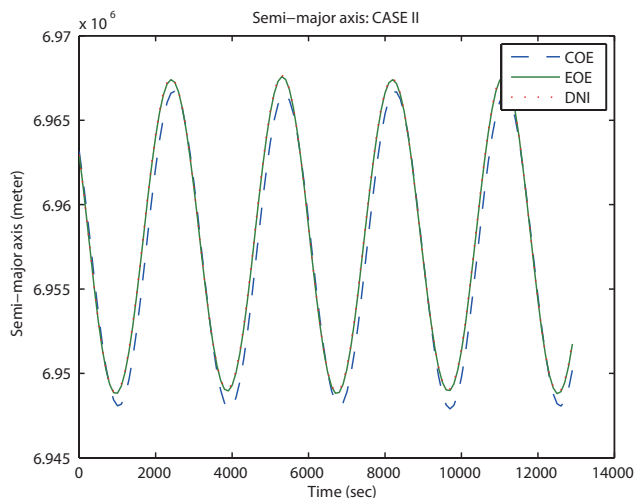

(a)

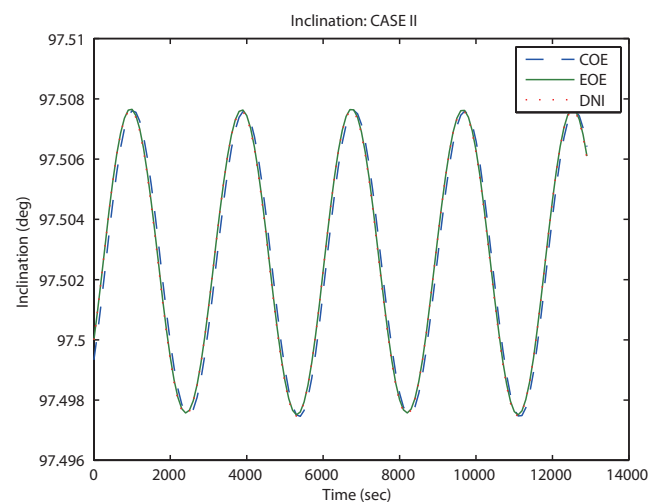

(c)

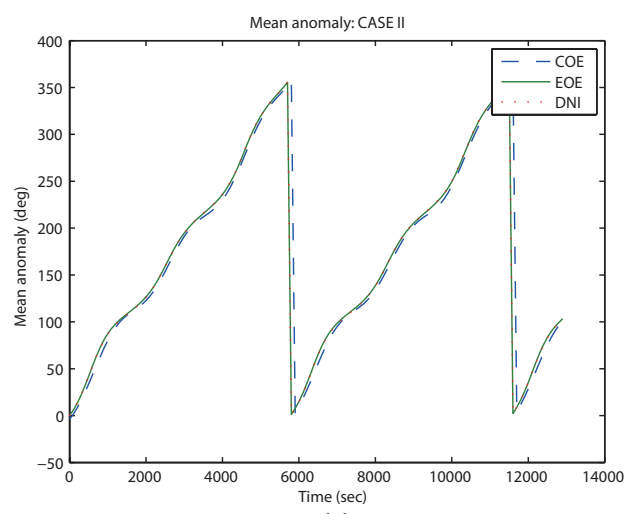

(e)

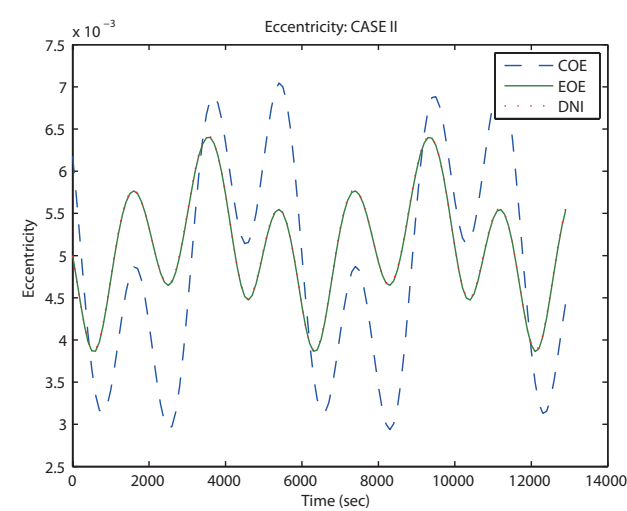

(b)

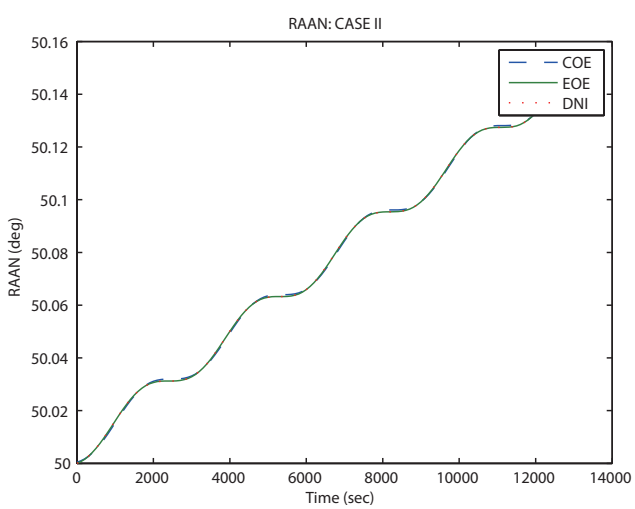

(d)

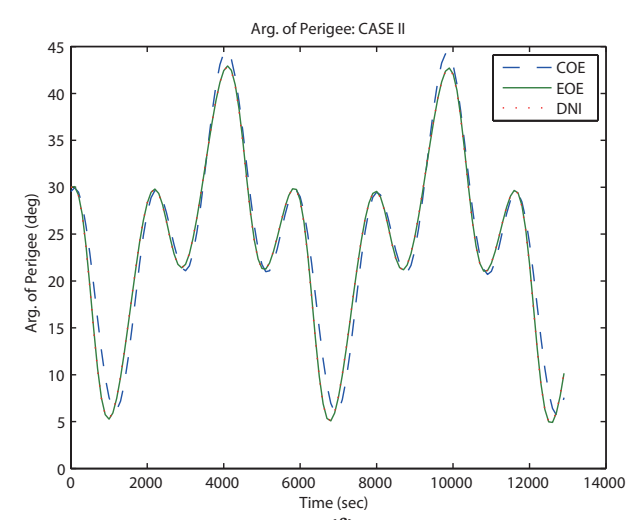

(f)

Fig. 8. The evolutions of orbital elements from two semi-analytical solutions and direct numerical integration (DNI) method with the $J_{2}$ perturbation for 13,000 seconds in case II. From left upper corner, (a) semi-major axis, (b) eccentricity, (c) inclination, (d) right ascension of ascending node, (e) mean anomaly, and (f) argument of perigee. Red line by COE LPE-DNI, blue line by EOE LPE-DNI. 
same magnitude of troubles. More rigorous analysis on the evolution of the eccentricity will be performed on a separate study.

The second comparison test was performed in same manner with one day duration to check the consistencies. Also, we can check the slight hint of long term error behavior of each orbital element from different solutions.
With the seven day duration of time, only few details of variation trend can survive.

In Fig. 12, you can find the various types of error evolutions of semi-major axis. With only $J_{2}$ perturbation, long period perturbation behavior was not expected. The orbital trajectory from each method (LPE with COE, LPE with EOE, and DNI) did not show obvious long periodic

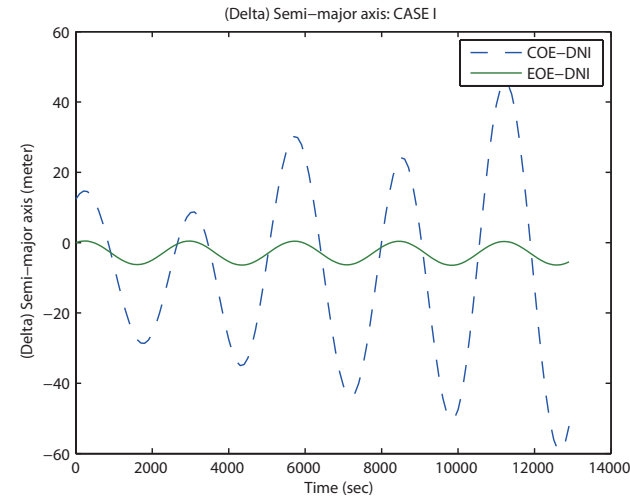

(a)

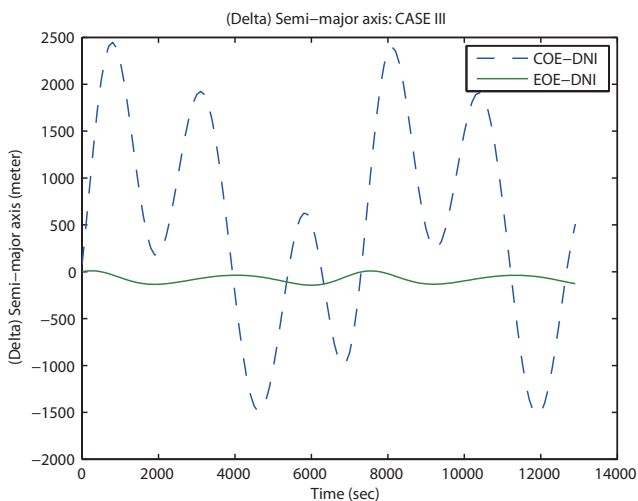

(c)

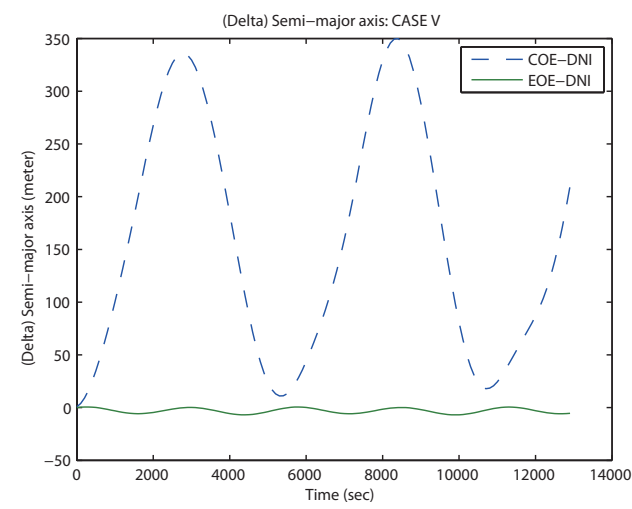

(e)

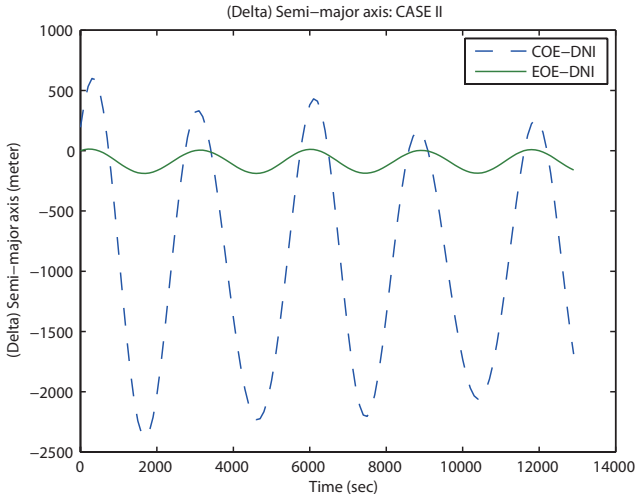

(b)

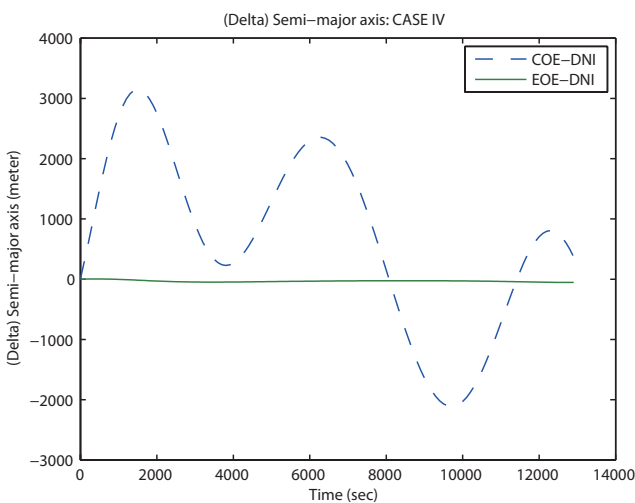

(d)

Fig. 9. The differences in semi-major axes of two semi-analytical solutions from direct numerical integration (DNI) result with the $J_{2}$ perturbation for all cases. Red line by COE LPE-DNI, blue line by EOE LPE-DNI. 
perturbed motion in seven days. However, the cases I, II, and $\mathrm{V}$ show periodic variation in errors semi-major axis as shown in Figs. 12a, b, and e. Also, you can see similar variation in right ascension of ascending node as in Figs 13a-c, and e. In case of argument of perigee, same kind of periodic variations can be seen in Figs. 14b and c. But it cannot be confirmed as a long period perturbation with- out further analysis.

Then the third comparison test was performed in same manner with seven day duration to check the long term variation in each element. With the seven day duration of time, only few details of variation trend can survive. However, the cases I and V show periodic variation in error evolutions in semi-major axis and right ascension of

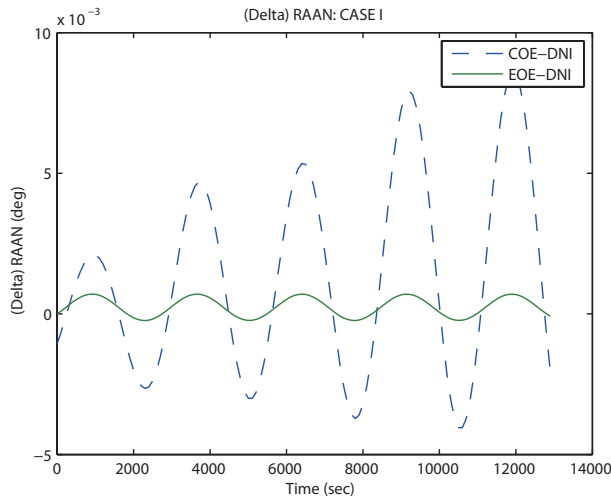

(a)

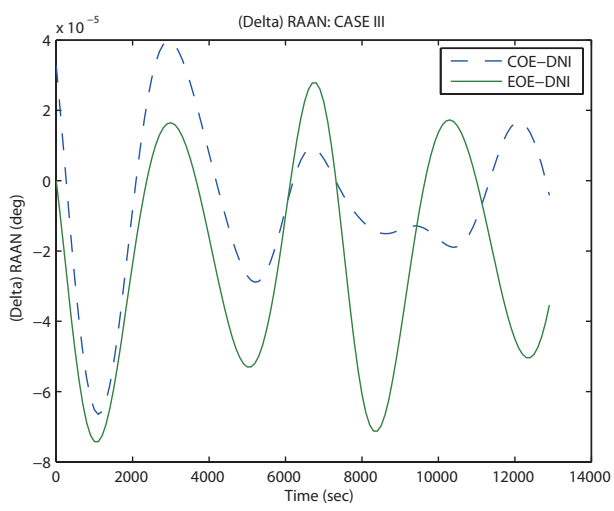

(c)

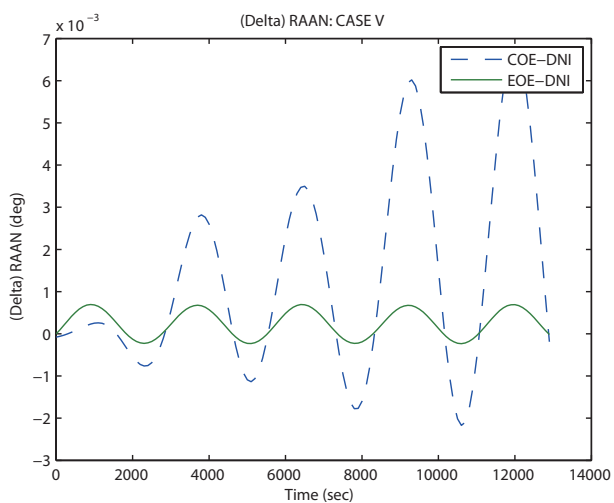

(e)

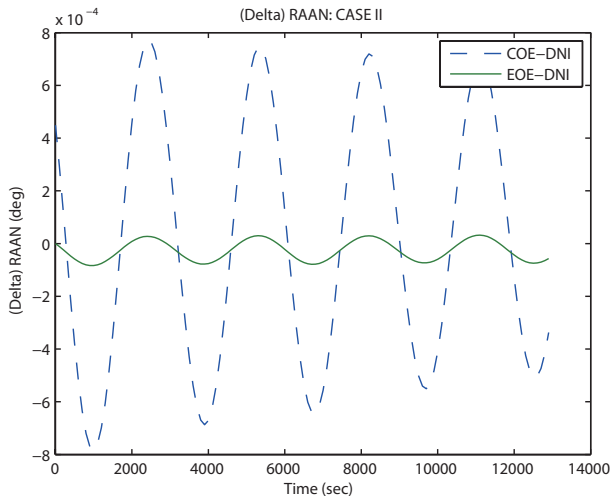

(b)

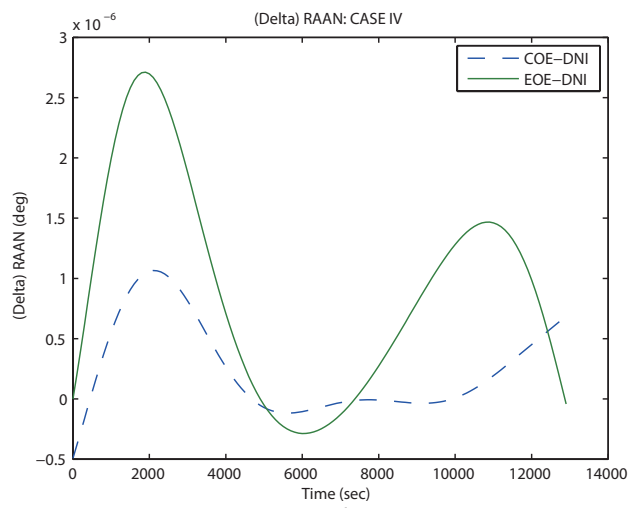

(d)

Fig. 10. The differences in right ascension of ascending nodes of two semi-analytical solutions from direct numerical integration (DNI) result with the $J_{2}$ perturbation for all cases. Red line by COE LPE-DNI, blue line by EOE LPE-DNI. 
ascending node as shown in Figs. 15a and e, 16a and e. Also, you can see secular like variation in semi-major axis as in Fig. 15d. The divergence trend can be seen in the error evolutions in semi-major axis as in Figs. 15b and c, in right ascension of ascending node as in Figs. 16b-d, in argument of perigee as in Figs. 17b-d.

In summary, the description of a perturbed motion

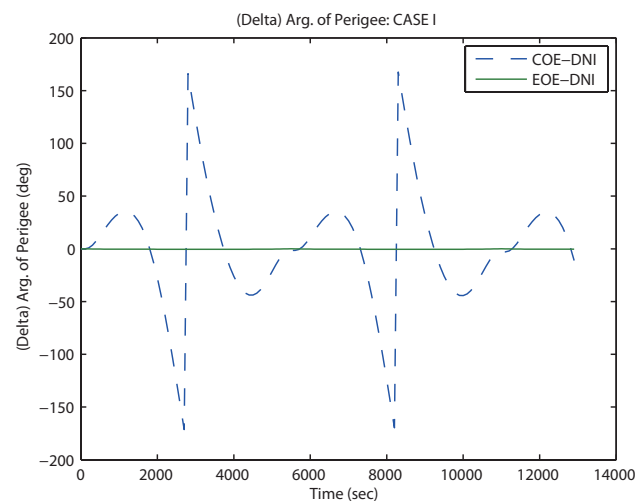

(a)

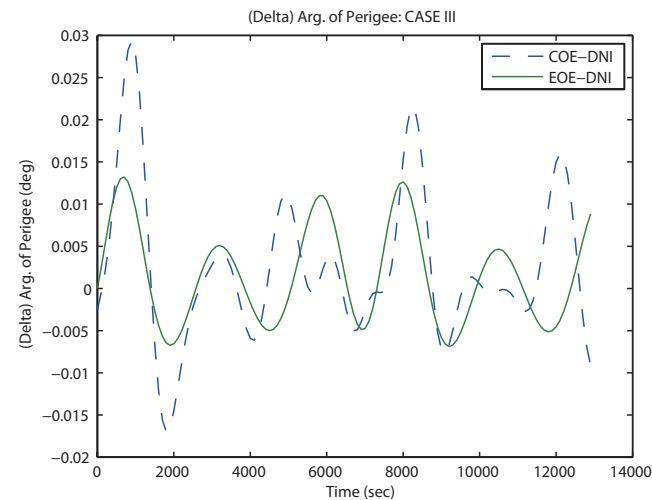

(c)

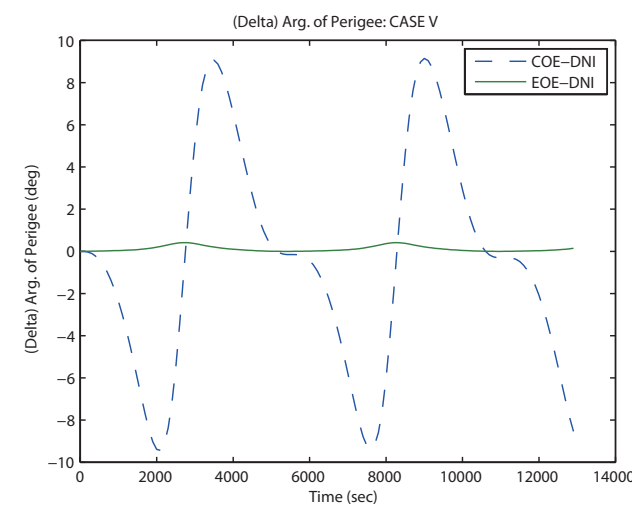

(e) of satellite by LPE with COE can be erratic on several orbital elements in specific cases like small eccentricity or small inclination. As you can see in most figures, EOE LPE agrees relatively better with DNI method than COE LPE does in most elements. It is consistent with different sizes (semi-major axis), shapes (eccentricity), and orbital plane position (inclination and right ascension of

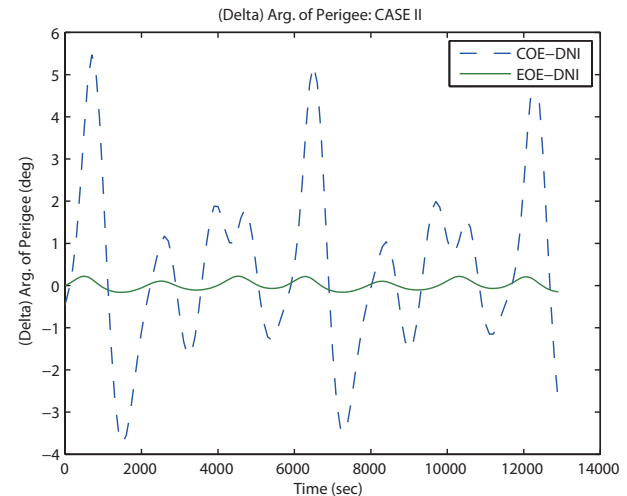

(b)

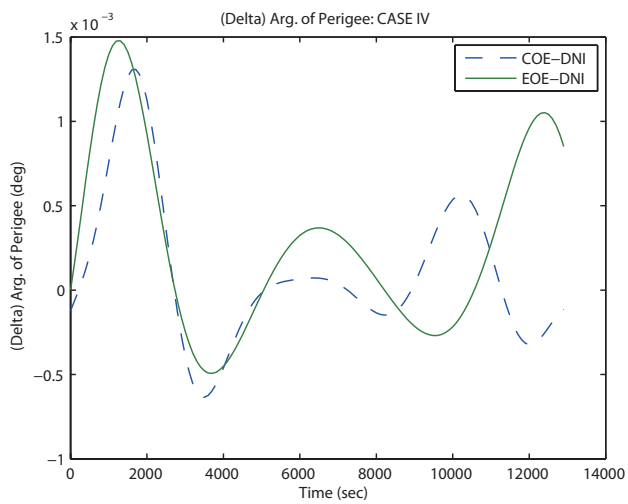

(d)

Fig. 11. The differences in arguments of perigees of two semi-analytical solutions from direct numerical integration (DNI) result with the $J_{2}$ perturbation for all cases. Red line by COE LPE-DNI, blue line by EOE LPE-DNI. 
ascending node).

In this paper, we selected only three elements, semimajor axis, right ascension of ascending node, and argument of perigee to present the error evolution by time. However, the position of a satellite on an orbit can be defined by mean anomaly. This non geometric angle plays an important role as a running variable instead of time in LPE with COE. In this study, the trends of variation in mean anomaly for each case shows similar pattern as in argument of perigee. In the same manner, (semi-major axis, eccentricity), (right ascension of ascending node, inclination), and (argument of perigee, mean anomaly) can be loosely couple by its similarity in the variations.

The theories and equations we used in this study are

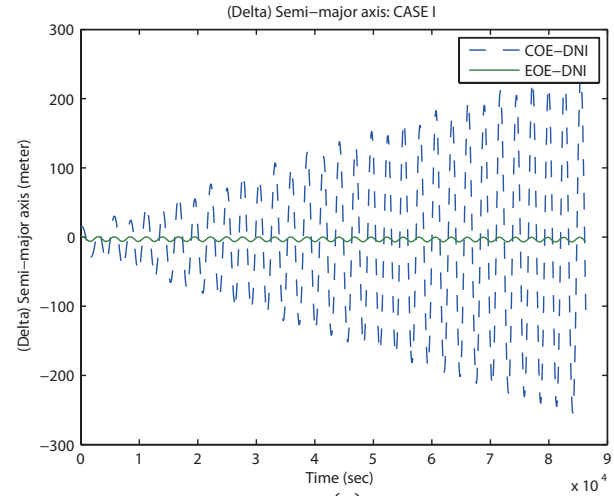

(a)

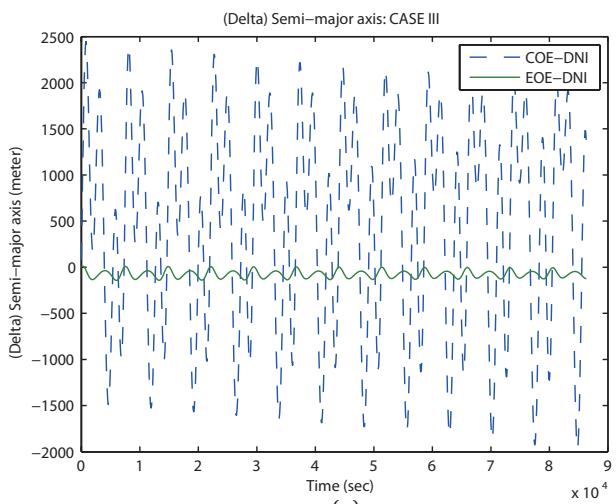

(c)

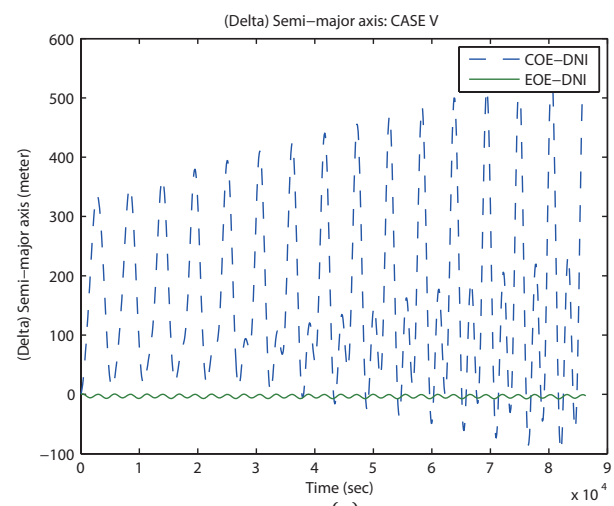

(e)

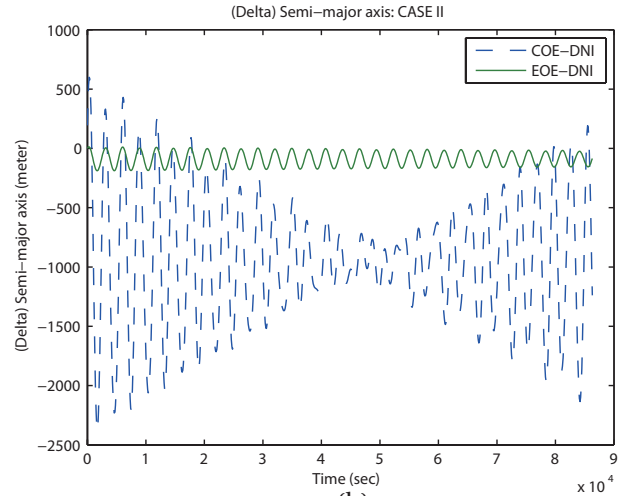

(b)

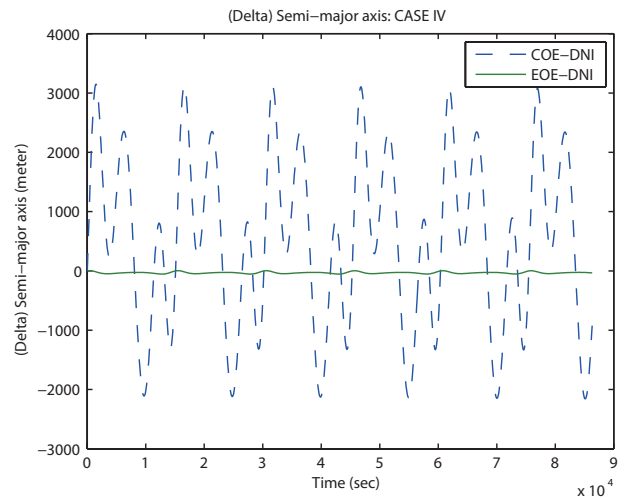

(d)

Fig. 12. The differences in semi-major axes of two semi-analytical solutions from direct numerical integration (DNI) result with the $J_{2}$ perturbation for all cases in one day. Red line by COE LPE-DNI, blue line by EOE LPE-DNI. 
consistent with Kozai (1959), Brouwer \& Clemence (1961) and Vallado (2004). And the result from EOE LPE and DNI agreed each other. Not until further study and analysis are performed, the long periodic variations in the solutions of COE LPE cannot be confirmed.

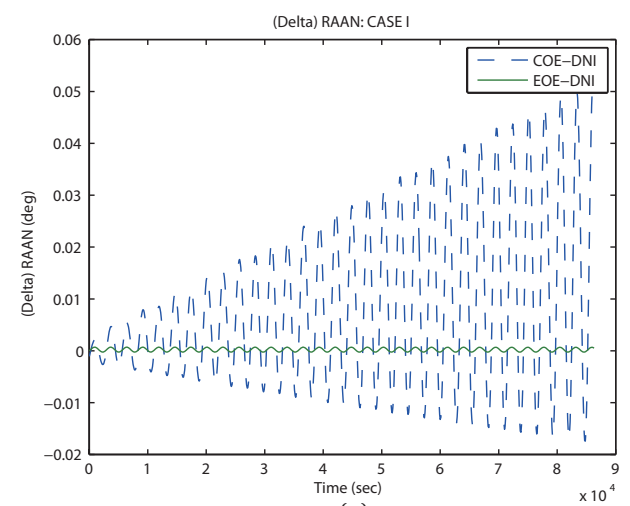

(a)

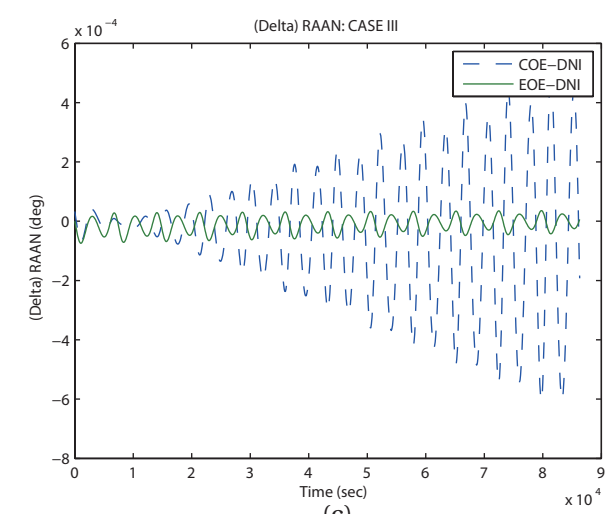

(c)

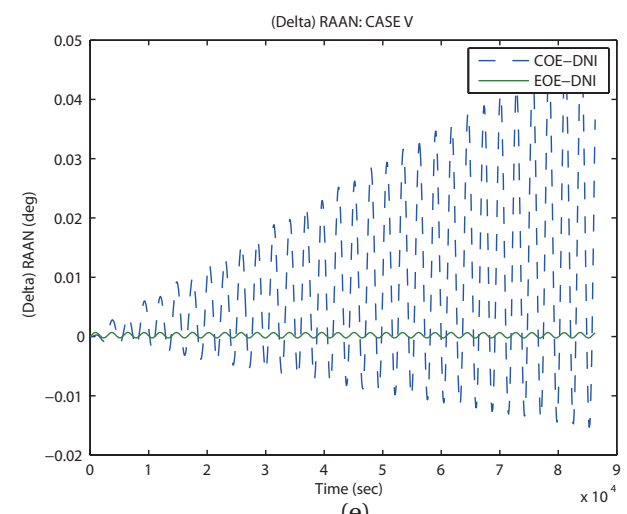

(e)

\section{CONCLUSIONS}

In this study, we compared the three methods to describe the motion of a satellite with the $J_{2}$ perturbation up to seven days. The time evolutions of orbital elements are presented to check the system stability with the several near singular conditions of the equations. As a result,

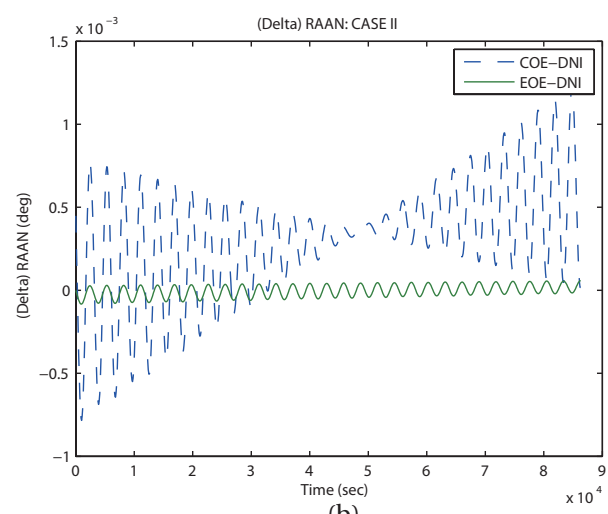

(b)

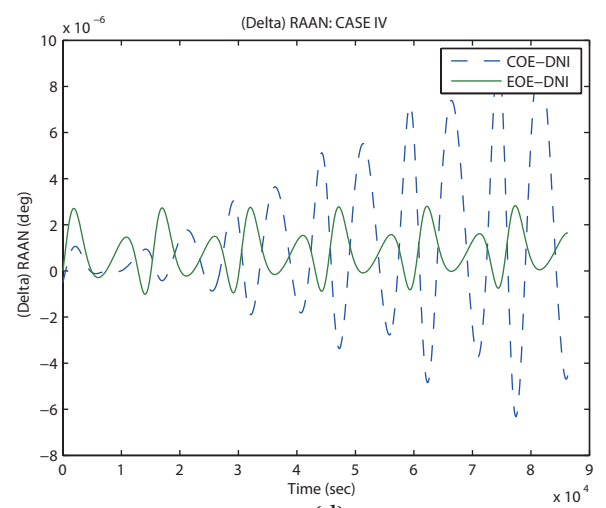

(d)

Fig. 13. The differences in right ascensions of ascending nodes of two semi-analytical solutions from direct numerical integration (DNI) result with the $J_{2}$ perturbation for all cases in one day. Red line by COE LPE-DNI, blue line by EOE LPE-DNI. 
Lagrange planetary equation with equinoctial orbital element agreed better with direct numerical integration method than Lagrange planetary equation with classical Kepler orbital elements in our test cases.
This study was partially supported by the project "GNSS measurement processing and analysis" internally funded by Korea Astronomy and Space Science Institute (KASI).

\section{ACKNOWLEDGEMENTS}

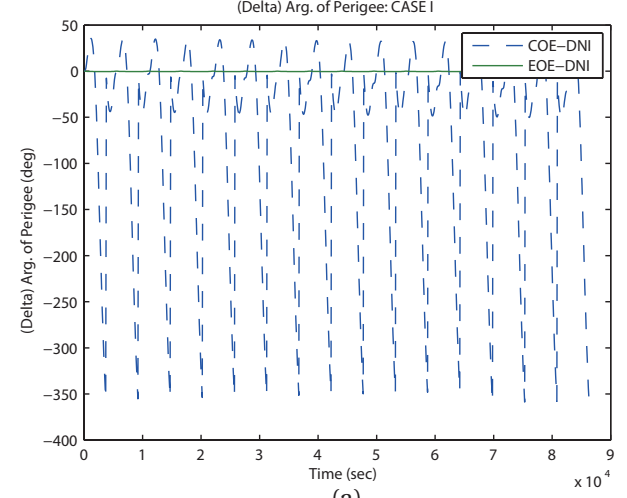

(a)

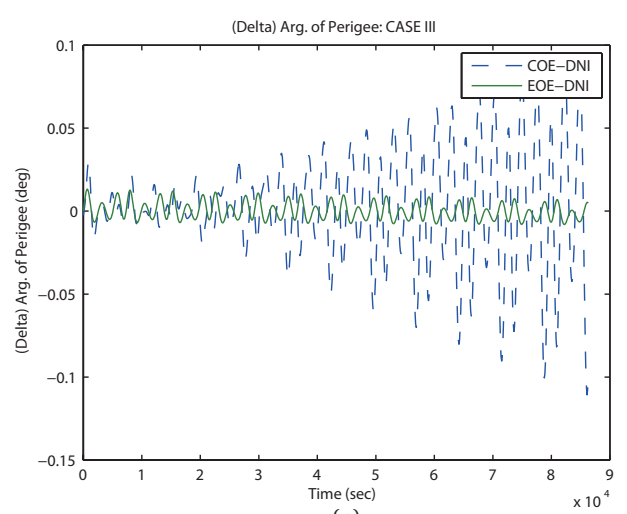

(c)

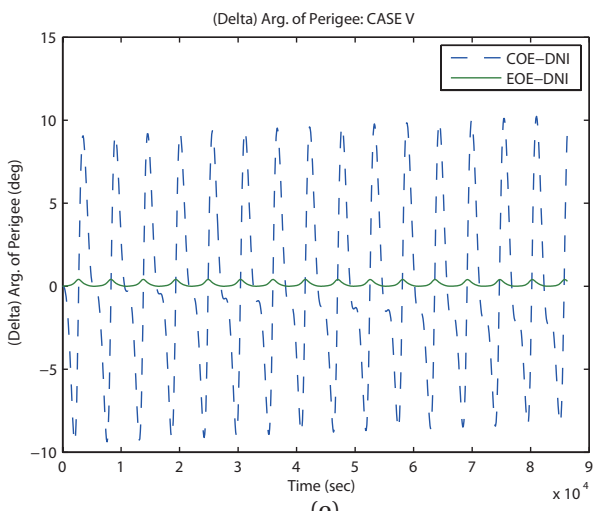

(e)

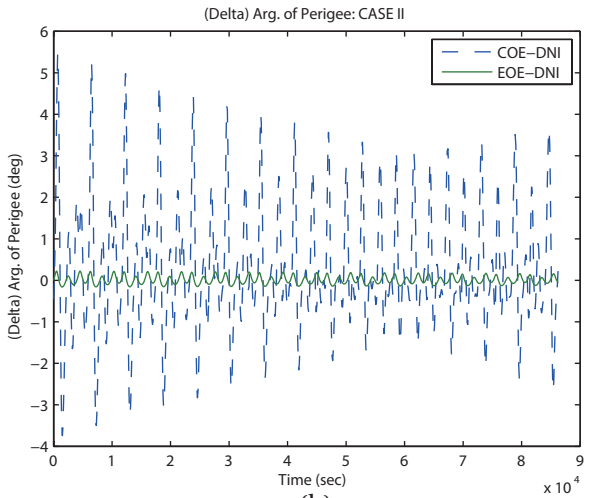

(b)

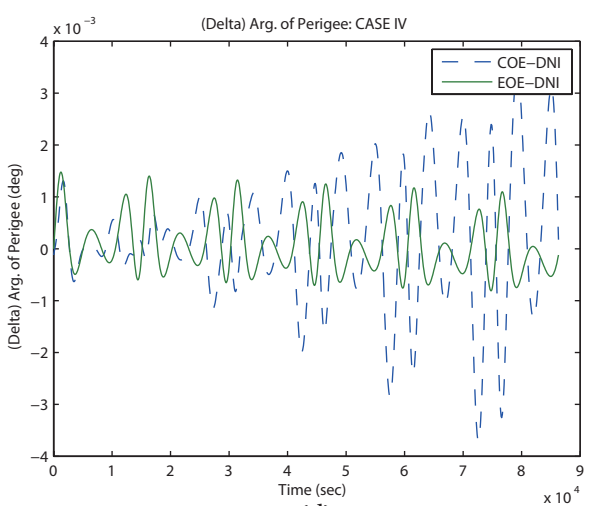

(d)

Fig. 14. The differences in arguments of perigees of two semi-analytical solutions from direct numerical integration (DNI) result with the $J_{2}$ perturbation for all cases in one day. Red line by COE LPE-DNI, blue line by EOE LPE-DNI. 


\section{REFERENCES}

Arsenault JL, Ford KC, Koskela PE, Orbit determination using analytic partial derivatives of perturbed motion, AIAAJ, 8, 4-12 (1970). doi: 10.2514/3.5597

Blitzer L, Handbook of orbital perturbation (University of Arizona Press, Tempe, 1975), 28-32.

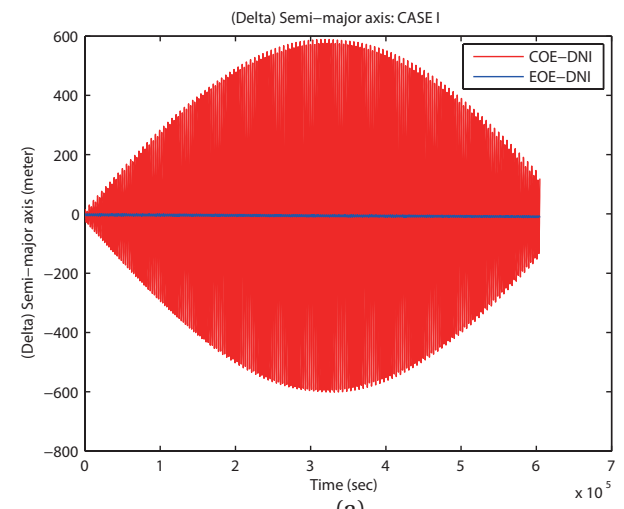

(a)

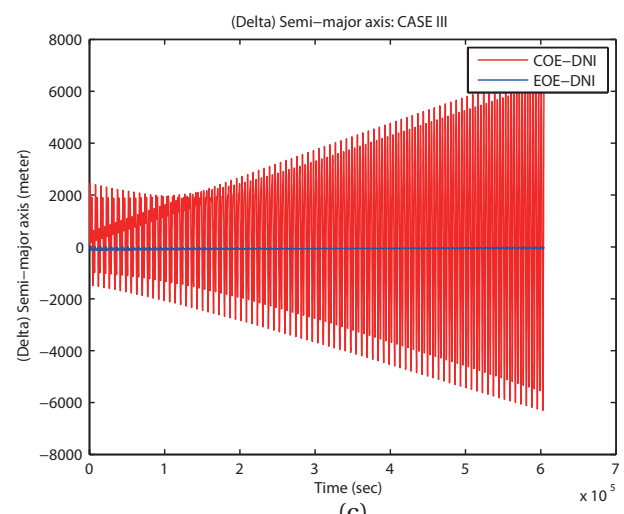

(c)

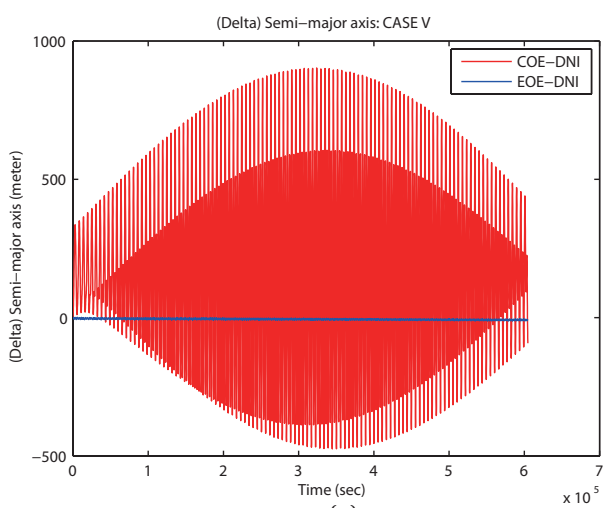

(e)
Broucke RA, Cefola PJ, On the equinoctial orbit elements, CeMec, 5, 303-310 (1972). doi: 10.1007/BF01228432 Brouwer D, Clemence GM, Methods of celestial mechanics (Academic Press, New York, 1961), 273-307.

Cohen CJ, Hubbard EC, A nonsingular set of orbit elements, AJ, 67, 10 (1962). doi: 10.1086/108597

$\mathrm{Jo} \mathrm{JH}$, The comparison of numerical integration methods for

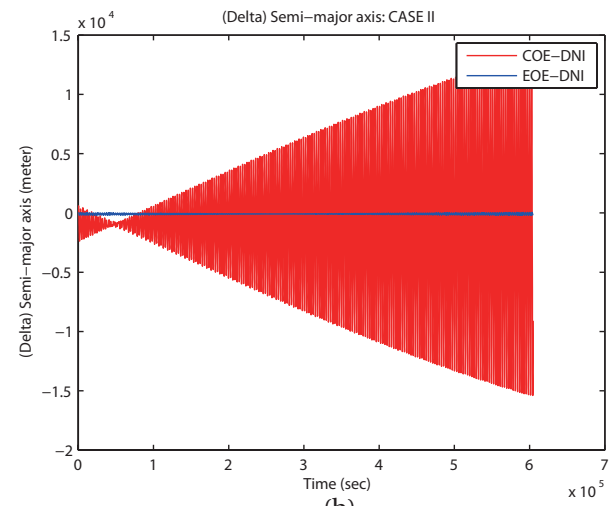

(b)

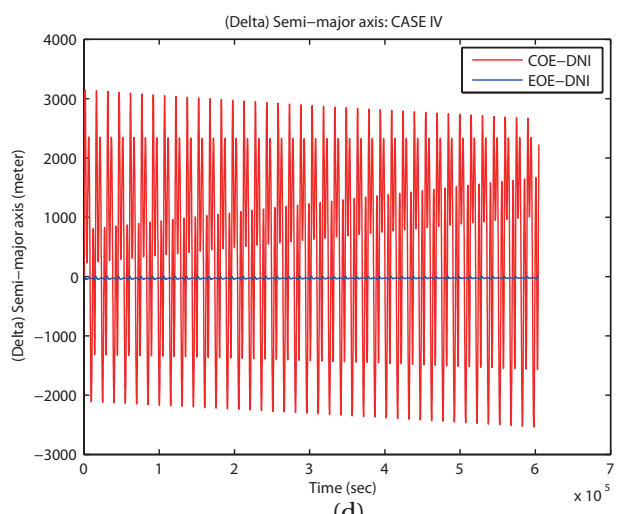

(d)

Fig. 15. The differences in semi-major axes of two semi-analytical solutions from direct numerical integration (DNI) result with the $J_{2}$ perturbation for all cases in seven days. Red line by COE LPE-DNI, blue line by EOE LPE-DNI. 
the KASIOPEA, Part II, Bull Korean Space Sci Soc, 17, 2627 (2008).

Kozai Y, The motion of a close earth satellite, AJ, 64, 367 (1959). doi: 10.1086/107957

Luthcke SB, Zelensky NP, Rowlands DD, Lemoine FG, Williams TA, The 1-centimeter Orbit: Jason-1 precision orbit determination using GPS, SLR, DORIS and altimeter data, Mar Geodes, 26, 399-421 (2003).

Moulton FR, An introduction to celestial mechanics (MacMillan Company, New York, 1914), 387-425.

Nerem RS, Chao BF, Au AY, Chan JC, Klosko SM, et al., Temporal variations of the Earth's gravitational field from satellite laser ranging to Lageos, GeoRL 20, 595-598 (1993). doi: 10.1029/93GL00169

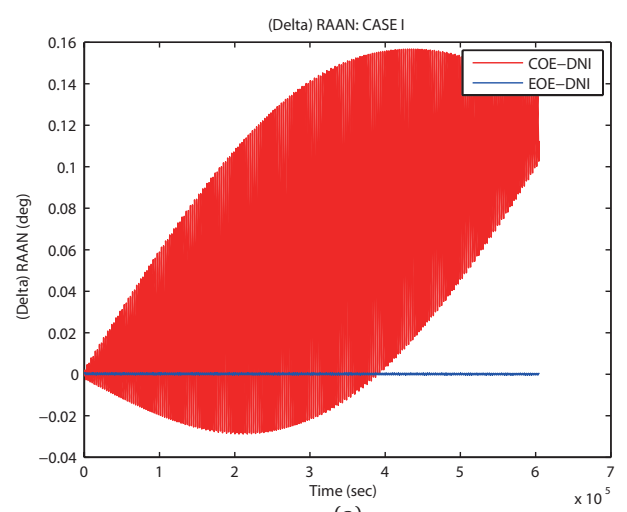

(a)

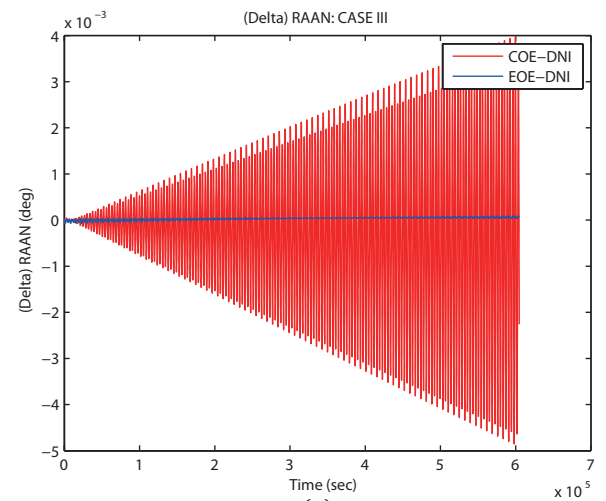

(c)

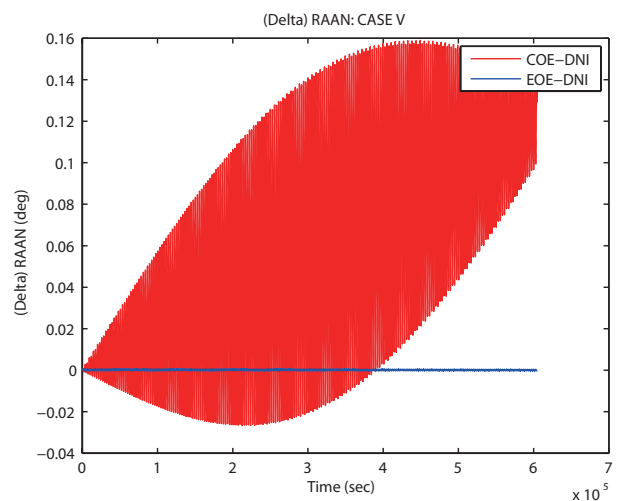

(e)

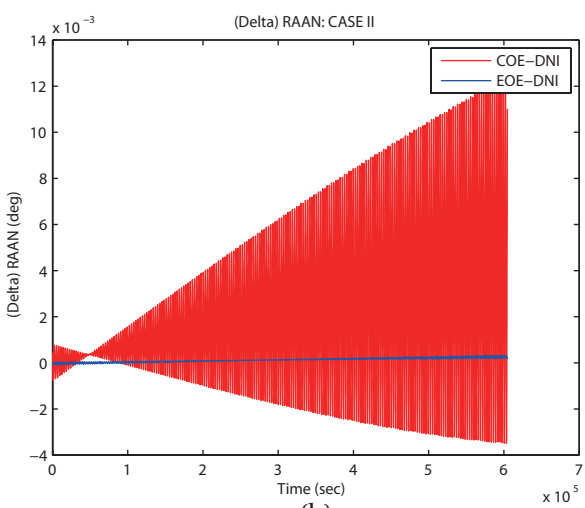

(b)

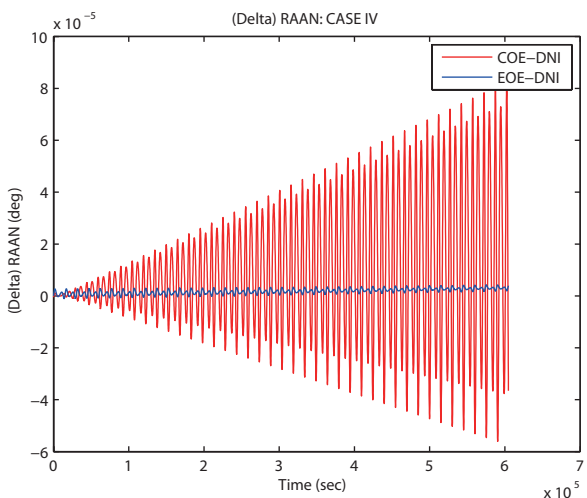

(d)

Fig. 16. The differences in right ascensions of ascending nodes of two semi-analytical solutions from direct numerical integration (DNI) result with the $J_{2}$ perturbation for all cases in seven days. Red line by COE LPE-DNI, blue line by EOE LPE-DNI. 
Taff LG, Celestial mechanics: a computational guide for the practitioner (Wiley, New York, 1985), 288-363.

Vallado DA, Fundamentals of astrodynamics and applications, 2nd ed. (Microcosm Press, El Segundo, CA, 2004), 491-674.
Walker MJH, Owens J, Ireland B, A set modified equinoctial orbit elements, CeMec, 36, 409-419 (1985). doi: 10.1007/ BF01227493

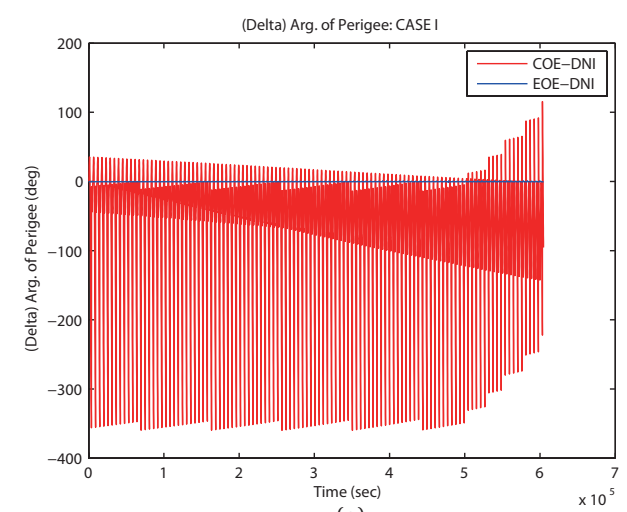

(a)

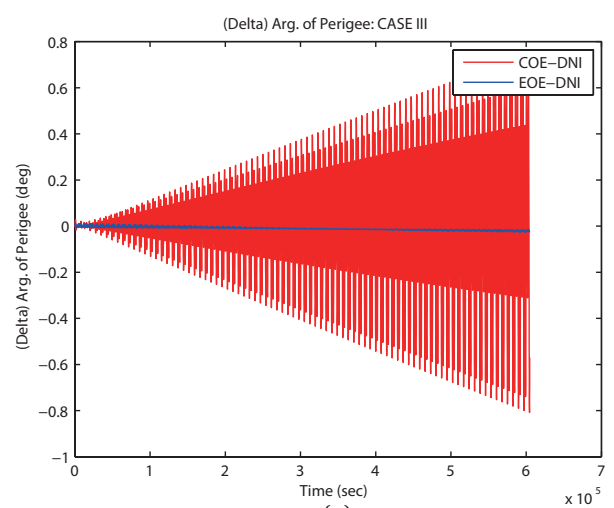

(c)

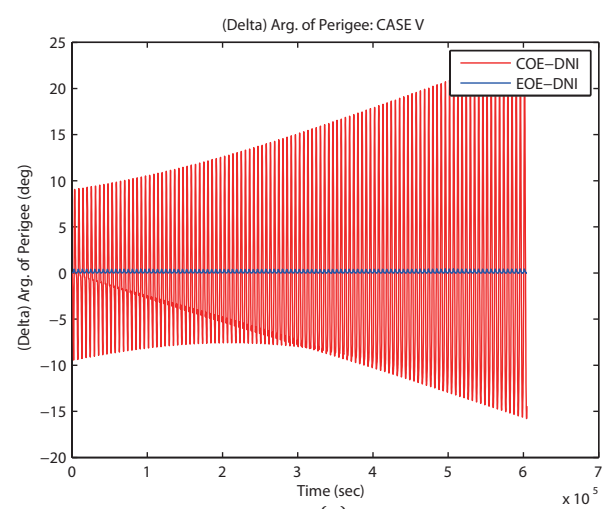

(e)

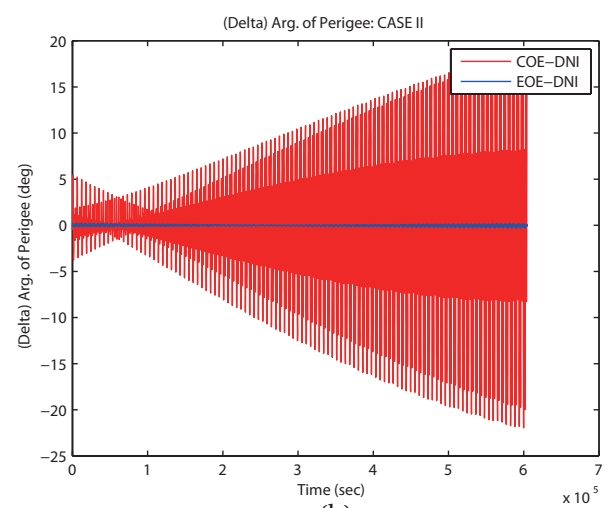

(b)

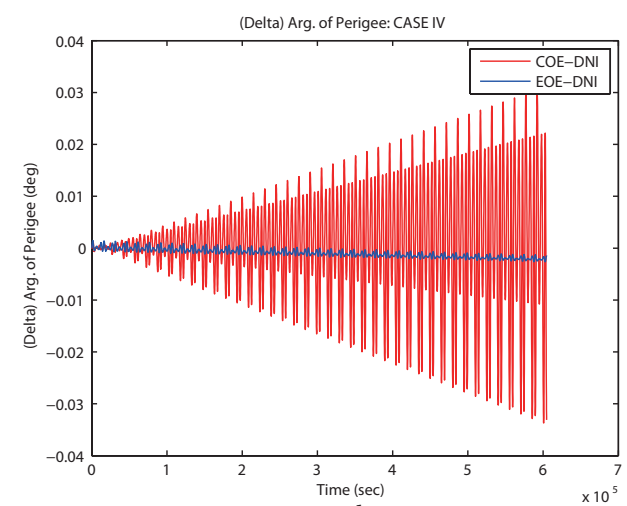

(d) for all cases in seven days. Red line by COE LPE-DNI, blue line by EOE LPE-DNI. 


\section{APPENDIX}

A. Short periodic perturbation on orbital elements by $J_{2}$

$$
\begin{aligned}
& A_{2}=\frac{3}{2} J_{2} R_{e}^{2} \\
& P=a\left(1-e^{2}\right) \\
& d a_{s}=\frac{A_{2}}{a}\left[\frac{2}{3}\left(1-\frac{3}{2} \sin ^{2} i\right)\left\{\left(\frac{a}{r}\right)^{3}-\left(1-e^{2}\right)^{-3 / 2}\right\}\right. \\
& \left.+\left(\frac{a}{r}\right)^{3} \sin ^{2} i \cos 2(v+\omega)\right] \\
& d e_{s}=\frac{1-e^{2}}{e} \frac{A_{2}}{a^{2}}\left[\frac{1}{3}\left(1-\frac{3}{2} \sin ^{2} i\right)\left\{\left(\frac{a}{r}\right)^{3}-\left(1-e^{2}\right)^{-3 / 2}\right\}\right. \\
& +\frac{1}{2}\left(\frac{a}{r}\right)^{3} \sin ^{2} i \cos 2(v+\omega)-\frac{\sin ^{2} i}{2 e} \frac{A_{2}}{a p}\{\cos 2 \\
& \left.\left.(v+\omega)+e \cos (v+2 \omega)+\frac{1}{3} e \cos (3 v+2 \omega)\right\}\right] \\
& d \omega_{s}=\frac{A_{2}}{p^{2}}\left[\left(2-\frac{5}{2} \sin ^{2} i\right)(v-M+e \sin v)+\left(1-\frac{3}{2} \sin ^{2} i\right)\right. \\
& \left\{\frac{1}{e}\left(1-\frac{1}{4} e^{2}\right) \sin v+\frac{1}{2} \sin 2 v+\frac{e}{12} \sin 3 v\right\} \\
& -\frac{1}{e}\left\{\frac{1}{4} \sin ^{2} i+\left(\frac{1}{2}-\frac{15}{16} \sin ^{2} i\right) e^{2}\right\} \sin (v+2 \omega) \\
& +\frac{e}{16} \sin ^{2} i \sin (v-2 \omega)-\frac{1}{2}\left(1-\frac{5}{2} \sin ^{2} i\right) \sin 2(v+\omega) \\
& +\frac{1}{e}\left\{\frac{7}{12} \sin ^{2} i-\frac{1}{6}\left(1-\frac{19}{8} \sin ^{2} i\right) e^{2}\right\} \sin (3 v+2 \omega) \\
& \left.+\frac{3}{8} \sin ^{2} i \sin (4 v+2 \omega)+\frac{e}{16} \sin ^{2} i \sin (5 v+2 \omega)\right] \\
& d i_{s}=\frac{1}{4} \frac{A_{2}}{p^{2}} \sin 2 i\{\cos 2(v+\omega)+e \cos (v+2 \omega) \\
& \left.+\frac{e}{3} \cos (3 v+2 \omega)\right\}
\end{aligned}
$$

$$
\begin{aligned}
d \Omega_{s}= & -\frac{A_{2}}{p^{2}} \cos i\left\{v-M+e \sin v-\frac{1}{2} \sin 2(v+\omega)\right. \\
& \left.-\frac{e}{2} \sin (v+2 \omega)-\frac{e}{6} \sin (3 v+2 \omega)\right\} \\
d M_{s}= & \frac{A_{2}}{p^{2}} \frac{\sqrt{1-e^{2}}}{e}\left[-\left(1-\frac{3}{2} \sin ^{2} i\right)\left\{\left(1-\frac{e^{2}}{4}\right)\right.\right. \\
& \left.\sin v+\frac{e}{2} \sin 2 v+\frac{e^{2}}{12} \sin 3 v\right\} \\
& +\sin ^{2} i\left\{\frac{1}{4}\left(1+\frac{5}{4} e^{2}\right) \sin (v+2 \omega)\right. \\
& -\frac{e^{2}}{16} \sin (v-2 \omega)-\frac{7}{12}\left(1-\frac{e^{2}}{28}\right) \\
& \quad \sin (3 v+2 \omega)-\frac{3}{8} e \sin (4 v+2 \omega) \\
& \left.\left.-\frac{e^{2}}{16} \sin (5 v+2 \omega)\right\}\right]
\end{aligned}
$$

B. Secular perturbation on orbital elements by the $J_{2}$

$$
\begin{aligned}
& \bar{\omega}=\omega_{0}+\frac{A_{2}}{p^{2}} \bar{n}\left(2-\frac{5}{2} \sin ^{2} i\right) t \\
& \bar{\Omega}=\Omega_{0}-\frac{A_{2}}{p^{2}} \bar{n} t \cos i \\
& \bar{M}=M_{0}+\bar{n} t \\
& \bar{n}=n_{0}+\frac{A_{2}}{p^{2}} n_{0}\left(1-\frac{3}{2} \sin ^{2} i\right) \sqrt{1-e^{2}} \\
& \bar{a}=a_{0}\left\{1-\frac{A_{2}}{p^{2}}\left(1-\frac{3}{2} \sin ^{2} i\right) \sqrt{1-e^{2}}\right\} \\
& \bar{n}^{2} \bar{a}^{3}=G M\left\{1-\frac{A_{2}}{p^{2}}\left(1-\frac{3}{2} \sin ^{2} i\right) \sqrt{1-e^{2}}\right\}
\end{aligned}
$$

Table A1. Initial condition in Cartesian coordinate.

\begin{tabular}{lrrrrr}
\hline & \multicolumn{1}{c}{ I } & \multicolumn{1}{c}{ II } & \multicolumn{1}{c}{ III } & \multicolumn{1}{c}{ IV } & \multicolumn{1}{c}{ V } \\
\hline$x_{0}(\mathrm{~m})$ & 3394660.36039 & 4203057.06462 & 1888283.89220 & -5322885.7156 & 3394660.36039 \\
$y_{0}(\mathrm{~m})$ & 5791071.41373 & 4305585.22888 & 6463054.22521 & 8804967.49923 & 5791071.41373 \\
$z_{0}(\mathrm{~m})$ & 585032.60546 & 3434432.91377 & 3793074.67830 & 5935450.57994 & 585032.605460 \\
$\dot{x}_{0}(\mathrm{~m} / \mathrm{s})$ & -6611.90492 & -1785.43259 & 558.63773 & 1359.87939 & -6626.75759 \\
$\dot{y}_{0}(\mathrm{~m} / \mathrm{s})$ & 3758.94652 & -3465.02071 & -3834.82817 & -2722.63785 & 3767.39044 \\
$\dot{z}_{0}(\mathrm{~m} / \mathrm{s})$ & 1156.93345 & 6528.94309 & 6256.09506 & 5258.44161 & 1159.53233 \\
\hline
\end{tabular}

